\title{
Corrigendum: Quantifying the shifting landscape of B cell immunodominance
}

Gordon A Dale, Jessica R Shartouny \& Joshy Jacob

Nat. Immunol. 18, 367-368 (2017); published online 22 March 2017; corrected after print 3 April 2017

In the version of this article initially published, a spelling error was made in the citation list. The error has been corrected in the HTML and PDF versions of the article.

\section{Erratum: Epigenetic landscapes reveal transcription factors that regulate $\mathrm{CD}^{+} \mathrm{T}$ cell differentiation}

Bingfei Yu, Kai Zhang, J Justin Milner, Clara Toma, Runqiang Chen, James P Scott-Browne, Renata M Pereira, Shane Crotty, John T Chang, Matthew E Pipkin, Wei Wang \& Ananda W Goldrath Nat. Immunol.; doi:10.1038/ni.3706; corrected online 27 March 2017

In the version of this article initially published online, some labels in Figure 2 were illegible or incorrect. Those should read "Enhancers $\left(\times 10^{3}\right)$ " along the top and "TE, MP and M" (top to bottom) along the left margin of Figure 2a; "N, TE, MP and M" (left to right) above the plot in Figure 2b; and "GO" below the plot in Figure 2c. Also, in the third sentence of the final paragraph of the final subsection of Results (Validation of PageRankpredicted TFs), the description of the control cells ("shCon-transfected") was incorrect. The correct text is "...lower among shNr3c1-transduced cells than among shCon-transduced cells.... The errors have been corrected in the print, PDF and HTML versions of this article.

\section{Erratum: THEMIS enhances TCR signaling and enables positive selection by selective inhibition of the phosphatase SHP-1}

Seeyoung Choi, Claude Warzecha, Ekaterina Zvezdova, Jan Lee, Jérémy Argenty, Renaud Lesourne, L Aravind \& Paul E Love Nat. Immunol.; doi:10.1038/ni.3692; corrected online 7 March 2017

In the version of this article initially published online, in the second sentence of the first paragraph of the third subsection of Results ('Deletion of Ptpn6 restores T cell development in Themis ${ }^{-1-}$ mice'), the TCR chain is identified incorrectly as 'CD3'; that phrase should read “....antibody to

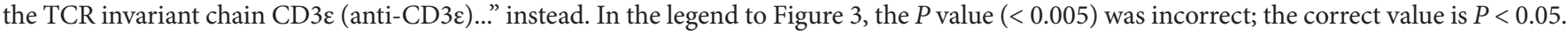
Also, in the final sentence of that legend, the directions '(left)' and '(right)' are incorrect; that should read "Data are representative of (top) or from (bottom) four experiments...” instead. In Figure 4a, the numbers along the horizontal axes are incorrectly vertical; they should be horizontal instead. In Figure 4b, the labels along the vertical axes of the second and fourth plots incorrectly include '(\%)'; the correct label is 'CD8SP cells $\left(\times 10^{6}\right)^{\prime}$ only. In the third sentence of the first paragraph of the fifth subsection of Results ('p-SHP-1 does not correspond with PTP activity'), the word 'of' is missing; this should read "The lower abundance of p-SHP-1..." instead. In the legend to Figure 5c, the antibody is incorrectly set off in commas; that should read “...immunoprecipitated with anti-SHP-1 from...” instead. Finally, Figure 7e is too large and should be the same size as all other panels in that figure. The errors have been corrected in the print, PDF and HTML versions of this article.

\section{Erratum: CCL19-CCR7-dependent reverse transendothelial migration of myeloid cells clears Chlamydia muridarum from the arterial intima}

Mark Roufaiel, Eric Gracey, Allan Siu, Su-Ning Zhu, Andrew Lau, Hisham Ibrahim, Marwan Althagafi, Kelly Tai, Sharon J Hyduk, Kateryna O Cybulsky, Sherine Ensan, Angela Li, Rickvinder Besla, Henry M Becker, Haiyan Xiao, Sanjiv A Luther, Robert D Inman, Clinton S Robbins, Jenny Jongstra-Bilen \& Myron I Cybulsky

Nat. Immunol. 11, 1263-1272 (2016); published online 26 September 2016; corrected after print 20 March 2017

In the version of this article initially published, the label along the horizontal axis of the graph in Figure 1a ('Dose (mg)') is incorrect. The correct label is 'Dose ( $\mu \mathrm{g})$ '. The error has been corrected in the HTML and PDF versions of the article. 